\title{
„Jetzt Sprichst Du!“ - Belastungen und psychosoziale Folgen der Corona-Pandemie für österreichische Kinder und Jugendliche
}

\author{
Manuel Schabus ${ }^{1,2} \&$ Esther-Sevil Eigl $\left.\right|^{1,2}$ \\ ${ }^{1}$ University of Salzburg; Department of Psychology; Laboratory for Sleep, Cognition and \\ Consciousness Research; Salzburg, Austria \\ ${ }^{2}$ University of Salzburg; Centre for Cognitive Neuroscience Salzburg (CCNS); Salzburg, Austria
}

Korrespondenzadresse:

Univ.-Prof. Dr. Manuel Schabus

Laboratory for Sleep, Cognition \& Consciousness Research

Hellbrunnerstraße 34

A-5020 Salzburg

Email: manuel.schabus@sbg.ac.at

Zusammenfassung: Die „Jetzt Sprichst Du!“-Umfrage veranschaulicht eindrücklich die psychosozialen Belastungen und die Beeinträchtigung von Kindern und Jugendlichen in Österreich während der aktuellen Corona-Pandemie. Im Rahmen einer Online-Umfrage wurden 5483 Kinder und Jugendliche im Alter von 6-18 Jahren bezüglich ihrer Gefühle, Ängste, Sorgen und Einschätzungen im Zusammenhang mit der Corona-Pandemie befragt.

6 Es zeigt sich, dass die Kinder und Jugendlichen durch die Situation geängstigt sind und 7 Mädchen hierbei über alle Altersgruppen hinweg stärker belastet sind. In diesem 8 Zusammenhang wird auch deutlich, dass das Risiko einer COVID-assoziierten 9 Hospitalisierung ähnlich wie bei Erwachsenen von den Kindern wie Jugendlichen massiv überschätzt wird. Auch macht sich in allen Altersgruppen eine hohe Perspektivenlosigkeit aufgrund der anhaltend herausfordernden Situation bemerkbar. Ferner häufen sich Gefühle von Wut, Ärger, Einsamkeit und Traurigkeit und es zeigt sich eine alarmierende Verschlechterung der Schlafqualität und eine Zunahme der Schlafproblematiken. Die Daten der „Jetzt Sprichst Du!“-Umfrage betonen die Notwendigkeit eines unabdingbaren und raschen Handelns, um sowohl die psychosozialen, entwicklungspsychologischen als auch gesundheitlichen Kollateralschäden in dieser jungen Altersgruppe einzudämmen, soweit dies heute noch möglich ist.

Schlüsselwörter: psychosoziale Beeinträchtigung, Kinder, Jugendliche, Corona-Pandemie, Kollateralschäden, psychische Gesundheit, Coronavirus

Abstract: The survey „Jetzt Sprichst Du!” evaluates the psychosocial burden and impairments of children and adolescents in Austria during the ongoing COVID-19 pandemic. Using an online-questionnaire, 5483 children and adolescents between 6 and 18 years shared their feelings, fears, worries and thoughts regarding the Coronavirus pandemic. Most of them report a high degree of fear due to the current situation, with especially female participants being under more emotional strain than their male counterparts. Associated to this, the risk of a COVID-19-associated hospitalization is strongly overestimated, as previously found in adults. 
In addition, an alarming lack of perspective during the ongoing pandemic is evident across all age groups including the youngest participants aged 6-10 years. Feelings of fury, anger, loneliness, and sadness are reported much more frequently than previously. Last but not least, our study shows an alarming reduction of sleep quality and a drastic increase in self-reported sleep problems already in this young population. The results of the "Jetzt Sprichst Du!" survey emphasize the need for immediate action in order to limit the collateral damage caused on the psychosocial, developmental and health dimension as far as this is still possible today.

Keywords: psychosocial burden, children and adolescents, Covid-19 pandemic, collateral damage, mental health, coronavirus

\section{Hintergrund}

Die "Jetzt Sprichst Du"-Umfrage wurde am 21.Februar 2021 an der Universität Salzburg ins Leben gerufen, nachdem die psychosozialen Kollateralschäden der CoronaPandemie und ihrer Maßnahmen zunehmend sichtbar wurden [1, 2]. Zudem ist es evident, dass gerade Kinder und Jugendliche von der Pandemie massiv beeinträchtigt [3] und in ihrer Entwicklung eingeschränkt werden [4] und ihre Stimmen bis dato wenig bis gar nicht gehört wurden [5].

Der Studie vorausgegangen war eine Umfrage zu Wissen und Einstellungen der Allgemeinbevölkerung hinsichtlich der Corona-Pandemie ab dem achtzehnten Lebensjahr ${ }^{1}$.

\section{Methode}

Konkret haben 5483 Kinder und Jugendliche zwischen 6 und 18 Jahren im Zeitraum vom 21.2.2021 bis 19.4.2021 an der österreichweiten „Jetzt Sprichst Du“-Umfrage ${ }^{2}$ der Universität Salzburg teilgenommen. Bekannt gemacht wurde die Umfrage über die österreichische Presseagentur (APA), den ORF, und diverse Organisationen, die mit Kindern und Jugendlichen arbeiten.

Zur Datenbereinigung wurde vor der Auswertung der Daten neben dem Alter auch ein Plausibilitäts-Check auf Datenintegrität durchgeführt. Im Speziellen wurde bei der Frage „Was denkst Du: Von 1000 Schülerinnen und Schülern, die so sind wie Du, wie viele davon werden in den nächsten 12 Monaten schwer an Corona erkranken und im Krankenhaus landen?" und der damit assoziierten Frage „Wie groß schätzt Du die Gefahr für Dich persönlich ein, wegen Corona ins Krankenhaus zu müssen?" geprüft, ob von Personen, die (i) angaben, dass max. 1 Fall (auf 1000) ins Krankenhaus muss, folgerichtig auch die subjektiv eingeschätzte Gefahr wegen Corona ins Krankenhaus zu müssen als "minimal" oder "gar nicht" eingestuft wurde; zudem wurden Personen, die (ii) angaben, dass mindestens 412 von 1000 (95tes Perzentil) Kindern hospitalisiert werden müssten, nur dann in die finale Auswertung eingeschlossen, wenn sie zugleich ihr subjektives Risiko einer Corona-bedingten Hospitalisierung in diesem Sinne auch als "sehr groß" oder "groß" einschätzten. Die endgültige Stichprobengröße nach dieser Daten-Selektion ergab 5008 Kinder und Jugendliche zwischen 6 und 18 Lebensjahren, die die Umfrage bis zur letzten Frage des allgemeinen Teils beantworteten. Ein zweiter Teil

\footnotetext{
1 Vgl. Medienberichte auf www.sleepscience.at

2 Der Fragebogen ist auf http://bit.Iy/JetztSprichstDu supplements im Detail abzurufen.
} 
der Umfrage konnte optional und zusätzlich ausgefüllt werden und umfasste Fragen zu Schlafgewohnheiten, Aktivitätsniveau und Smartphone-Gebrauch. Diesen Teil beendeten 2232 Kinder und Jugendliche. Er wird in einer gesonderten Veröffentlichung im Detail behandelt [6].

Bei der Auswertung der Fragen mit Rangreihen als Antwortoption wurden in der Analyse die Häufigkeiten der ersten drei genannten Kategorien summiert, bzw. angegeben. Wenn als Rang 1 bereits eine Kategorie gewählt wurde, die andeutete, dass keine weiteren Optionen zutreffend waren (z.B: „Ich habe eigentlich keine Angst"), wurde nur der erste Rang berichtet.

\section{Resultate}

Von den 5008 Kindern und Jugendlichen waren 60,9\% der Teilnehmer:innen weiblich, 37,9\% männlich und 1,2\% divers. Die Stichprobe wurde in drei Altersgruppen eingeteilt: 6-10jährige „Volksschüler:innen“ ( $n=949), 11-14$-jährige „Mittelschüler:innen“ ( $n=1930)$ und 15-18jährige Jugendliche $(n=2129)$.

Auf die Frage, ob den Kindern und Jugendlichen „die aktuelle Situation mit Corona Angst" mache, antworten 48,1\% der Mädchen und 35,9\% der Jungen, dass ihnen die Situation „sehr" oder „ein bisschen“ Angst mache. Zwischen den Altersgruppen fällt auf, dass vor allem die Volksschüler die größten Ängste in Bezug auf Corona aufweisen und sich jedes zweite Kind durch die aktuelle Situation („sehr“ oder „ein bisschen“) verängstigt fühlt (für Details siehe Abb.1).

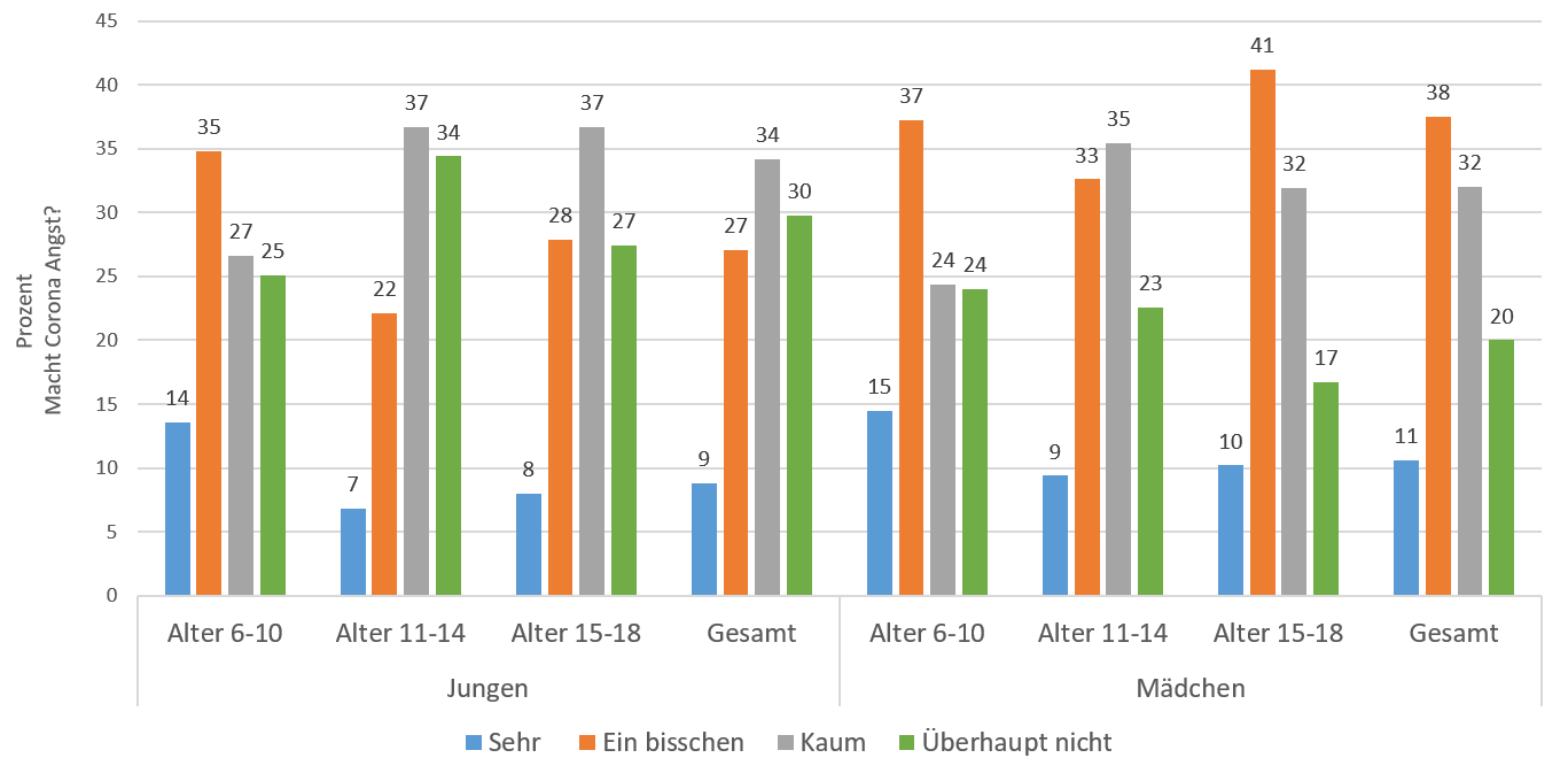

Abbildung 1. Verteilung der Antworten der Kinder und Jugendlichen auf die Frage "Macht Dir die aktuelle Situation mit Corona Angst?" nach Altersgruppen und Geschlecht.

Frägt man nach der Art dieser Ängste, so werden in erster Linie folgende Befürchtungen genannt: „Dass es noch lange dauern wird, bis das Leben so wie vorher wird“ $(54,4 \%)$, „dass das Leben gar nicht mehr so wie vorher wird“ $(50,1 \%)$, „dass Eltern, Geschwister oder nahe Angehörige sterben“ könnten (48,1\%) sowie dass sie „nicht mehr 
dieselben Zukunftschancen bzw. Jobmöglichkeiten“ wie „vor Corona“ haben könnten (36,8\%); nur $8,8 \%$ der Kinder und Jugendlichen gaben an, eigentlich keine dieser Ängste zu haben. Besonders bei den Jugendlichen ist die Angst, nicht mehr dieselben Zukunftschancen zu haben, deutlich ausgeprägt (47,8\%; vgl. Abb. 2$)$.

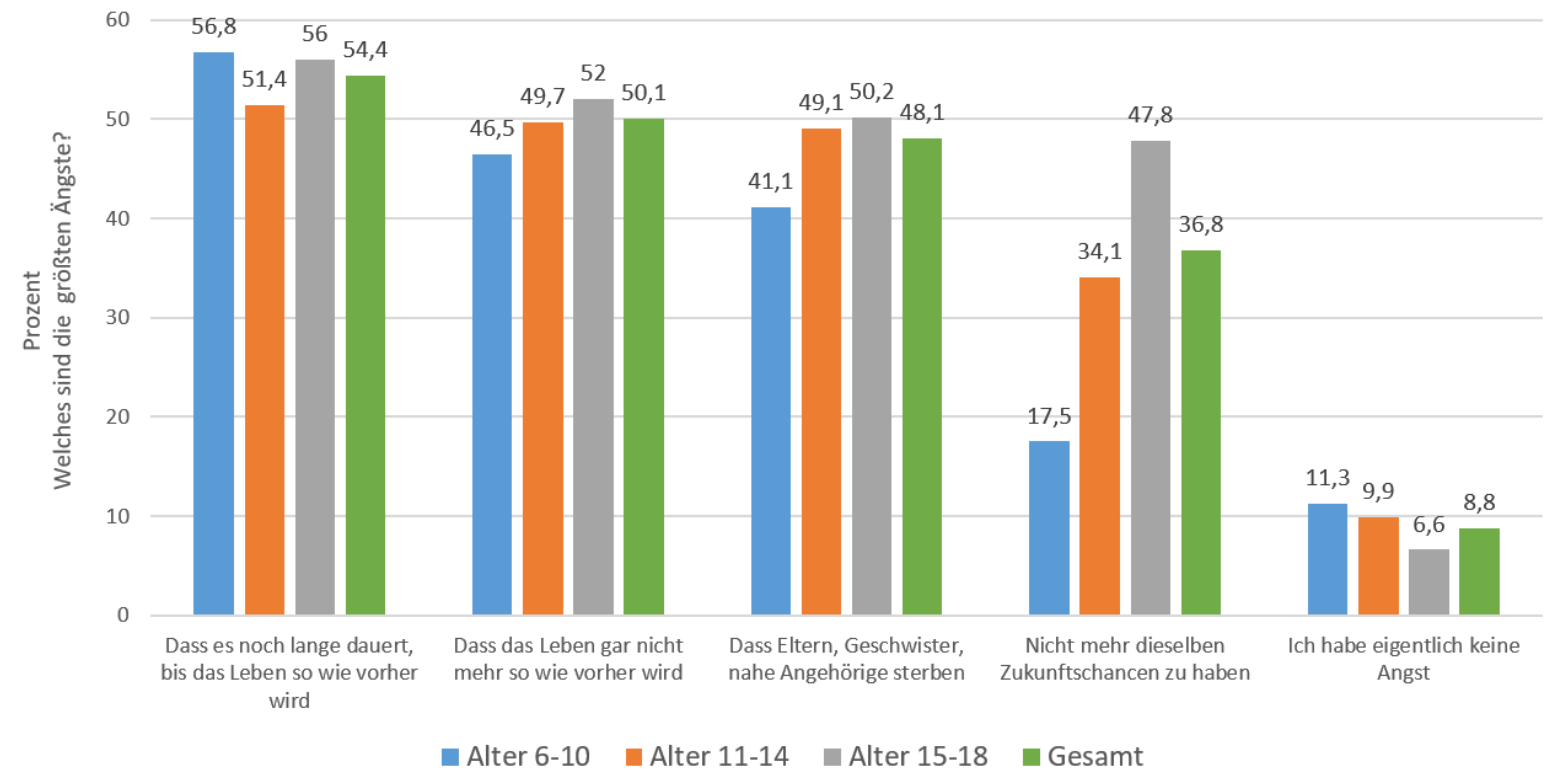

Abbildung 2. Verteilung der Antworten der Kinder und Jugendlichen auf die Frage "Wovor hast Du auf Corona bezogen - am meisten Angst?" nach Altersgruppen.

Als nächstes stellten wir uns die Frage, woher die Kinder und Jugendlichen hauptsächlich ihre Information über Corona beziehen. Auffällig ist, dass Volksschüler ihre Informationen bezüglich Corona primär über ihre Eltern und ihre Familie $(67,3 \%)$ beziehen, wohingegen Jugendliche primär „Social Media“ oder das Internet als Informationsquelle nutzen (46,9\%); öffentliches Fernsehen und Schule spielen eine vergleichsweise untergeordnete Rolle (vgl. Abb. 3).

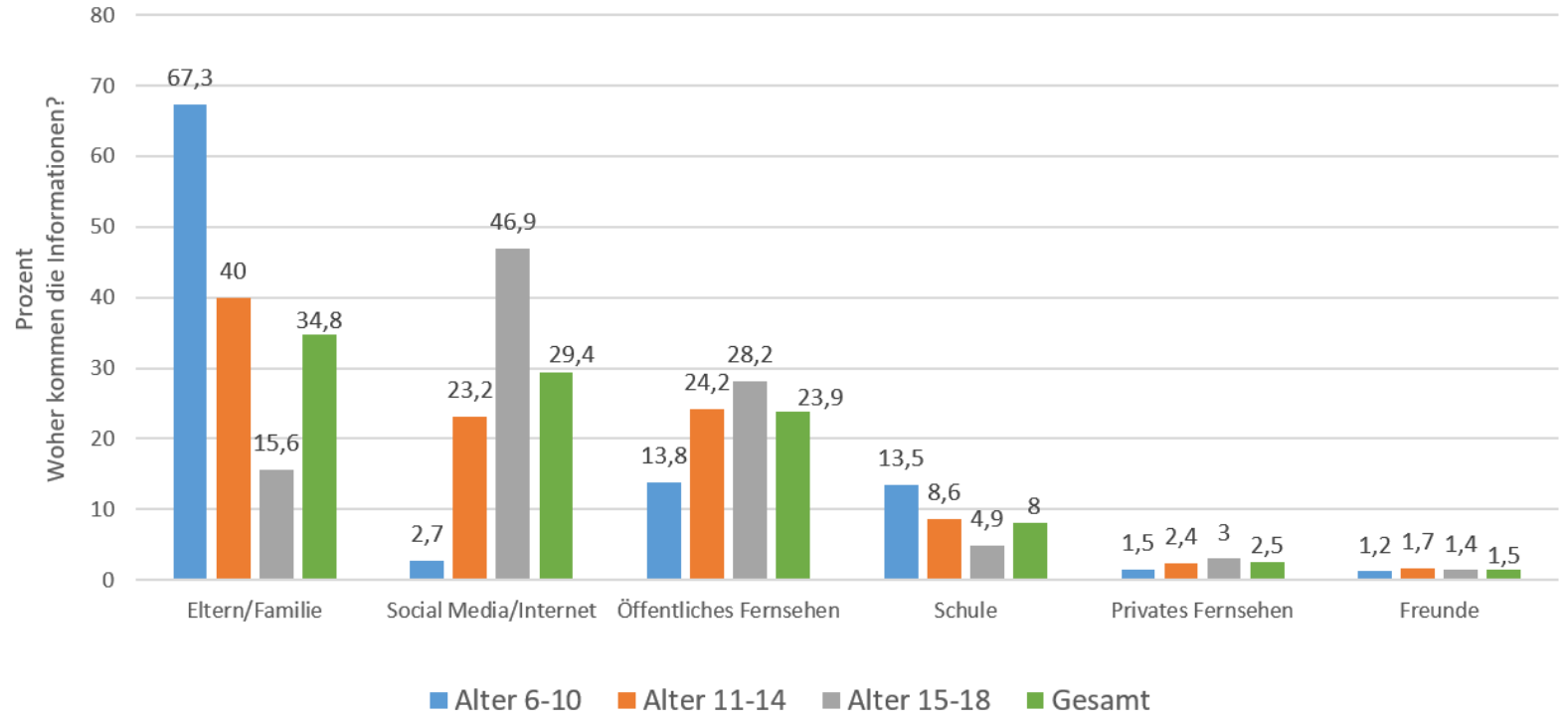

Abbildung 3. Verteilung der Antworten der Kinder und Jugendlichen auf die Frage "Woher bekommst Du hauptsächlich Deine Informationen über Corona?" nach Altersgruppen. 
Frägt man die Kinder und Jugendlichen, wie es ihnen im Vergleich zu vor Corona geht, so geben $71,8 \%$ an, dass es innen „viel“ $(25 \%)$ oder "ein bisschen“ $(46,8 \%)$ schlechter gehe als vor Corona (vgl. Abb. 4A). Frägt man, wann die Kinder und Jugendlichen denken, dass das „Leben wieder einigermaßen ,normal" sein wird“, so sagen $75,6 \%$, dass sie eine Rückkehr zur Normalität erst im Jahr 2022 oder später erwarten (vgl. Abb. 4B). Dies ist Ausdruck einer gewissen Perspektivenlosigkeit der Kinder und Jugendlichen, welche über alle Altersklassen beobachtet werden kann.

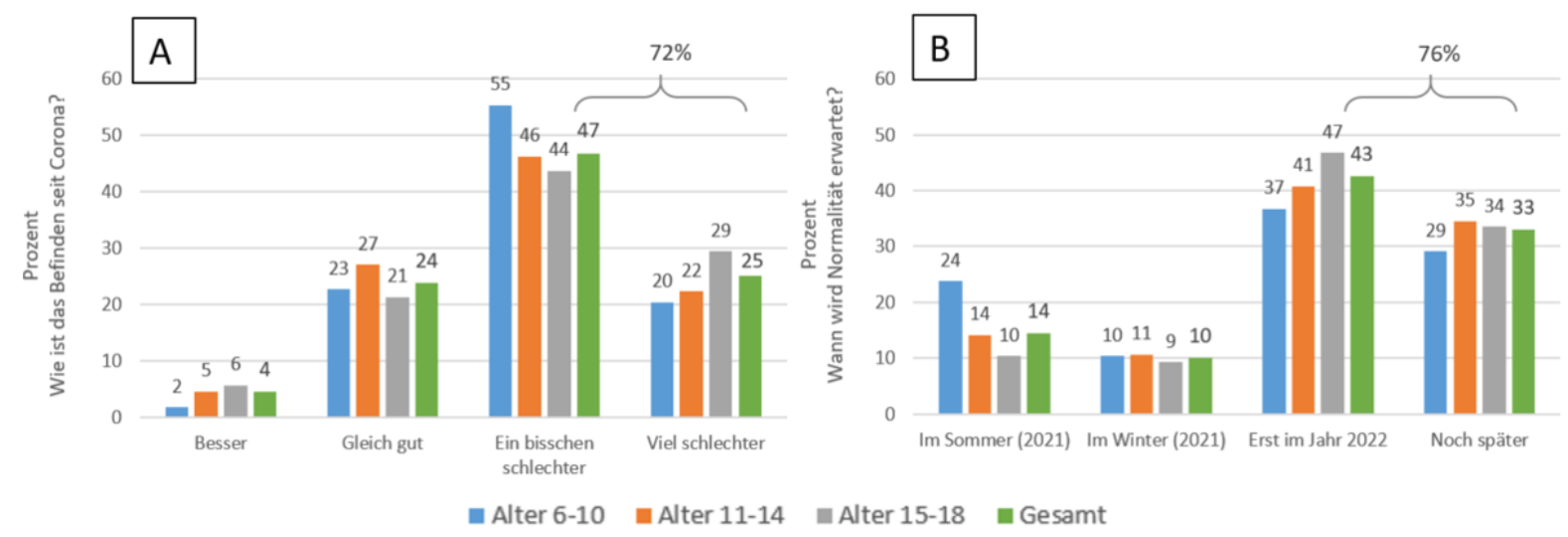

Abbildung 4 Verteilung der Antworten der Kinder und Jugendlichen auf die Frage A) „Wie geht es Dir im Vergleich zu vor Corona?" und B) „Was meinst Du, wann wird das Leben wieder einigermaßen "normal" sein?" nach Altersgruppen.

Ferner wurde nach den vorherrschenden Gefühlen seit Corona gefragt. Am häufigsten genannt wurde seit Corona „öfter wütend und genervt zu sein“ (58,2\%), gefolgt von „öfter einsam und allein zu sein“ (46\%) und „öfter traurig zu sein (42,7\%)“. 15,6\% fühlen sich „trotz Corona gut“ (13,6\%) oder „sogar besser“ (2\%; vgl. Abb. 5A). Frägt man nach den Dingen, die den Kindern und Jugendlichen von der Normalität am meisten fehlen, so wird am häufigsten angegeben „Freunde ohne Einschränkungen treffen zu können“ $(71,4 \%)$, gefolgt von „keine Masken tragen zu müssen und die Gesichter der Menschen sehen zu können“ (58,7\%), und „Sport" treiben zu können (41,4\%). Die Jugendlichen geben „Fortgehen“ als zweithäufigste Antwortoption (58,7\%) an (vgl. Abb. 5B). Über alle Altersgruppen hinweg berichten die Kinder und Jugendlichen, dass innen der normale Schulalltag „extrem“ $(29,8 \%)$ bzw. „ziemlich“ $(31,7 \%)$ abgeht. Vor allem die Volksschüler leiden hier am meisten unter der aktuellen Situation mit 72,2\% die angeben, dass innen der Alltag „sehr“ (42,1\%) oder „ziemlich“ (30,1\%) fehlt. 


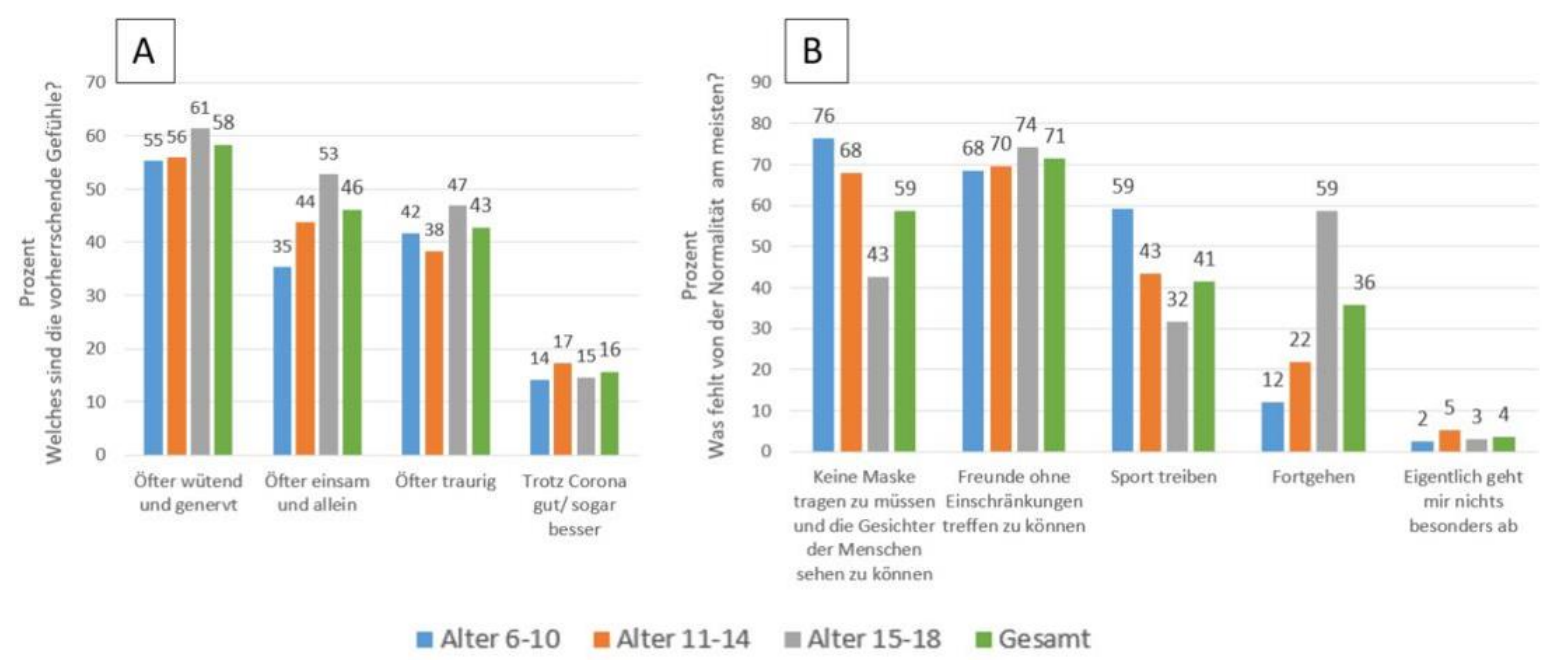

Abbildung 5. Verteilung der Antworten der Kinder und Jugendlichen auf die Frage A) "Wie fühlst Du Dich seit Corona?" und B) "Was geht Dir von der Normalität am meisten ab?" nach Altersgruppen

Laut Einschätzung der Kinder und Jugendlichen könne ihnen in der aktuellen Situation am meisten helfen „Freunde persönlich zu treffen“ (50,4\%), „Zeit mit der Familie $(49,2 \%)$ und „Zeit in der Natur" (39,3\%) zu verbringen. 4,4\% geben an, dass innen eigentlich aktuell nichts hilft.

Ferner wurden die Kinder und Jugendlichen nach ihrer Einschätzung des Risikos, das vom Corona-Virus (SARS-CoV-2) ausgeht, gefragt: „Was denkst Du: Von 1000 Schülerinnen und Schülern, die so sind wie Du, wie viele davon werden in den nächsten 12 Monaten schwer an Corona erkranken und im Krankenhaus landen?". Interessanterweise wird das Risiko wegen einer SARS-CoV-2-Infektion ins Krankenhaus zu müssen von Kindern und Jugendlichen massiv überschätzt und mit 1,2\%-3,3\% beziffert, obwohl das Risiko in dieser jungen Altersgruppe unter 1 in $10.000(<0,01 \%)$ Menschen mit Risikofaktoren [7,8] bzw. unter 1 in 40.000 (<0.003\%) bei Kindern/Jugendlichen ohne Risikofaktoren liegt [7] (vgl. Tabelle 1).

Tabelle 1. Geschätztes Risiko der Kinder und Jugendlichen wegen einer SARS-CoV-2Infektion ins Krankenhaus zu müssen

\begin{tabular}{llll} 
& Alter 6-10 & Alter 11-14 & Alter 15-18 \\
\hline 5\% getrimmtes Mittel & $12(1,2 \%)$ & $33(3,3 \%)$ & $31(3,1 \%)$ \\
\hline $\begin{array}{l}\text { QCovid Risikobewertung (https://qcovid.org/) } \\
\text { für eine COVID-19-assozierte Hospitalisierung bei einer } \\
\text { 19-jährigen, männlichen Person }\end{array}$ & $\begin{array}{l}1 \text { in } 43478(0,0023 \%) \text { ohne Risikofaktoren } \\
1 \text { in } 10753(0,093 \%)\end{array}$ & mit Diabetes Typ 1 und Asthma
\end{tabular}

Anmerkung: Der QCovid® Algorithmus der Universität Oxford (UK) ist ein evidenz-basiertes Modell, welches Faktoren wie Alter, Geschlecht, Ethnizität oder Vorerkrankungen einbezieht, um das Risiko einer COVID-19assoziierten Hospitalisierung oder eines durch COVID-19 verursachten Todes abzuschätzen. Unter https://qcovid.org kann dieses Risiko für jedes Individuum errechnet werden. Als Veranschauungsbeispiel ist in Tabelle 1 das Risiko einer 19-jährigen, männlichen Person $(180 \mathrm{~cm}, 80 \mathrm{~kg})$ ohne Vorerkrankungen bzw. mit Diabetes Typ 1 und Asthma dargestellt und kann als obere Grenze verstanden werden. 
Auch die Angst, dass ein Elternteil oder naher Angehöriger stirbt - welche eine der der primären Ängste der Kinder und Jugendlichen ist (Abb. 2) - scheint stark überhöht, nachdem das tatsächliche Risiko einer Hospitalisierung It. QCovid® selbst für die in den Medien oft strapazierten „durchschnittlichen Großeltern“ (Modell gerechnet mit einer 75-jährigen Frau mit Diabetes Typ 2, Asthma, $165 \mathrm{~cm}, 70 \mathrm{~kg})$ bei ca. 1 in 469 Fällen $(0,21 \%)$ und das des Versterbens bei ca. 1 in 1344 (0,07\%) Fällen liegt.

191

Außerdem haben wir versucht zu verstehen, ob es einen Unterschied macht, ob Kinder und Jugendliche in einem Umfeld mit auf Corona bezogen ängstlichen oder wenig ängstlichen Eltern leben. Hierfür wurde die Variable "Was denken Deine Eltern über Corona?" zur Kategorisierung herangezogen und Kinder und Jugendliche, welche der Meinung waren, die Eltern hielten Corona für „sehr gefährlich“ oder „gefährlich“ wurden der „ängstlichen“ Gruppe zugeordnet. Kinder, welche angaben, dass ihre Eltern Corona als „überhaupt nicht gefährlich“, „nicht sehr gefährlich“ oder „ähnlich gefährlich wie eine Grippe“ einschätzten, wurden als „nicht ängstliche" Gruppe definiert.

Über die Altersgruppen hinweg zeigt sich, dass die ängstliche Gruppe häufiger angab, ihre „Verwandten fast gar nicht mehr gesehen zu haben“ $(44,6 \%$ vs. $33,6 \%$ bei der nichtängstlichen Gruppe), oder etwas mehr Angst vor dem Virus zu haben (47,1\% vs. 40,1\%). Bei der Einschätzung der Gefährlichkeit des SARS-CoV-2 Erregers gab die ängstliche Gruppe zu $84 \%$ an, Corona sei „sehr gefährlich“ oder "gefährlich“, wobei nur 13\% der nicht-ängstlichen Gruppe dieser Einschätzung folgten. Auch schätzten 3x mehr Kinder der ängstlichen Gruppe die Gefahr eines COVID-assoziierten Krankenhausaufenthaltes als (sehr) groß ein (15,2\% vs. 4,7\%). Ein ähnliches Bild der Überschätzung des Risikos für eine Hospitalisierung ergibt sich im Rahmen einer Abfrage in absoluten Zahlen, wenngleich in einem anderen Ausmaß $(4,28 \%$ vs. $1,52 \%$ Personen, „die genauso sind wie du“).

Auch die größten Ängste, die von den Kindern und Jugendlichen angegeben werden, unterscheiden sich in diesen beiden Gruppen maßgeblich. Wo die ängstliche Gruppe als primäre Befürchtung angibt, dass „Eltern, Geschwister oder nahe Angehörige sterben“ könnten (61\%) bzw. „dass Eltern, Geschwister oder nahe Angehörige erkranken“ könnten (42,6\%; vgl. Abb.6A), beschäftigt die nicht-ängstliche Gruppe vor allem, dass „das Leben gar nicht mehr so wie vorher" werden könnte $(61,1 \%)$, bzw. dass sie „nicht mehr die dieselben Zukunftschancen bzw. Jobmöglichkeiten“ wie vor Corona haben könnten (44,8\%; vgl. Abb. $6 B)$.

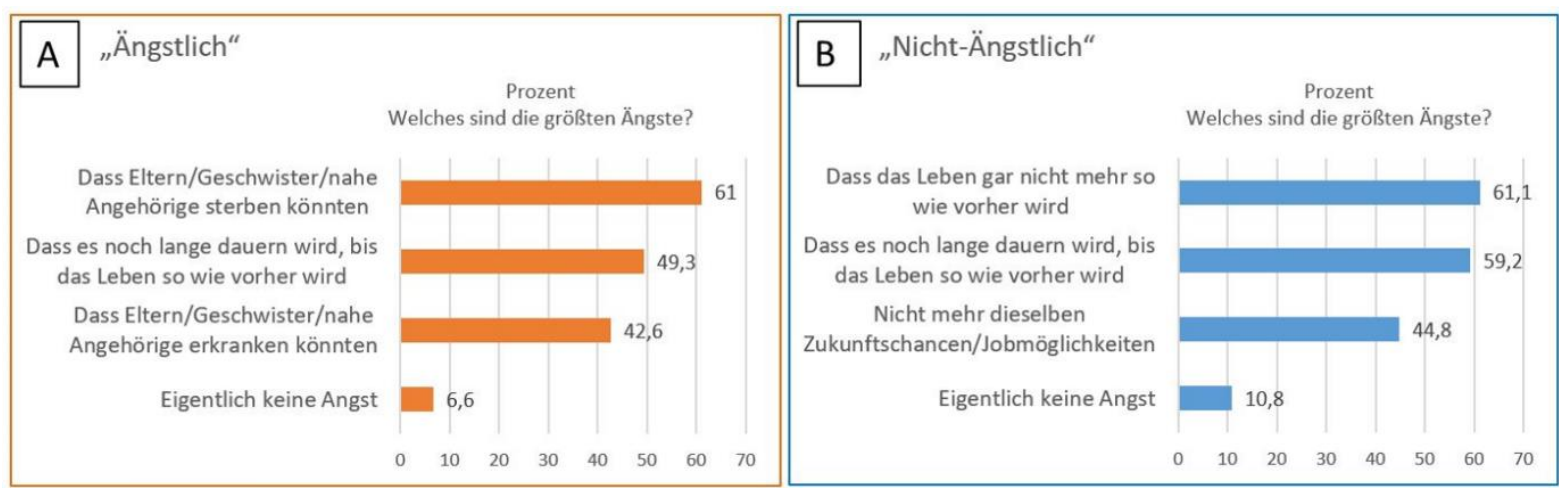

Abbildung 6. Verteilung der Antworten der Kinder und Jugendlichen auf die Frage "Wovor hast Du auf Corona bezogen - am meisten Angst?" in der Gruppe des A) "ängstlichen" vs. B) "nicht- 
ängstlichen" familiären Umfelds. Die Gruppe wurde nach den (von den Kindern/Jugendlichen) eingeschätzten Ängsten und Sorgen der Eltern gebildet.

Vergleicht man diese beiden Gruppen nach ihren vorherrschenden Gefühlen seit Corona, zeigt sich das überraschende Bild, dass es die Kinder und Jugendlichen der nichtängstlichen Gruppe sind, die häufiger angeben, sich seit Corona „öfter wütend und genervt“ zu fühlen (63,5\% vs. 52,6\%). Vermutlich ist es für diese Kinder schwer nachzuvollziehen, weshalb sie im Schulalltag eine Maske tragen müssen, Selbsttests durchführen sollen oder weshalb strenge Distanzregeln gelten und die somit einem erhöhten Druck im System ausgesetzt sind. Zudem zeigt sich, dass das Gefühl, sich „öfter einsam und allein zu fühlen“ vom Volksschulalter bis hin zur Gruppe der Jugendlichen merkbar ansteigt. Hier liegt die Vermutung nahe, dass dies damit zusammenhängt, dass es die Corona-Maßnahmen den Jugendlichen nicht oder nur bedingt erlauben, ihre Freunde und Altersgenossen zu treffen, wohingegen Volksschulkinder ohnehin noch mehr Zeit in der Familie selbst verbringen (vgl. Tabelle 2).

Tabelle 2. Vorherrschende Gefühle seit Corona in den Gruppen (Corona-) "ängstlich“ vs. „nicht-ängstlich“.

\begin{tabular}{|lcccc|}
\hline „Ängstlich“ & Gesamt & Alter 6-10 & Alter 11-14 & Alter 15-18 \\
\hline & $n=2.456$ & $n=383$ & $n=922$ & $n=1.151$ \\
\hline Ich bin öfter traurig als vor Corona & 39,8 & 37,6 & 36,6 & 43,0 \\
\hline Ich fühle mich öfter einsam und allein & 44,9 & 34,1 & 42,6 & 50,5 \\
\hline Ich fühle mich öfter wütend und genervt & 52,6 & 43,8 & 49,8 & 57,9 \\
\hline Ich fühle mich trotz Corona gut oder sogar besser* & 19,4 & 19,6 & 21,0 & 18,1 \\
\hline & & & & \\
\hline "Nicht-Ängstlich“ & Gesamt & Alter 6-10 & Alter 11-14 & Alter 15-18 \\
\hline & $n=2.546$ & $n=566$ & $n=1.004$ & $n=976$ \\
\hline Ich bin öfter traurig als vor Corona & 45,4 & 44,4 & 40,0 & 51,4 \\
\hline Ich fühle mich öfter einsam und allein & 47,1 & 36 & 45,0 & 55,5 \\
\hline Ich fühle mich öfter wütend und genervt & 63,5 & 63,1 & 61,7 & 65,6 \\
\hline Ich fühle mich trotz Corona gut oder sogar besser* & 12,0 & 10,6 & 13,9 & 10,7 \\
\hline
\end{tabular}

Anmerkung: *Akkumulierung der Antwortoptionen „Ich fühle mich trotz Corona gut" und "Ich fühle mich sogar besser als zuvor".

Im zweiten Teil des Fragebogens, der hier nur exemplarisch behandelt wird ( $n=2232)$, fällt auf, dass die körperlichen Aktivitäten seit Corona bei 74,8\% der Kinder und Jugendlichen mit „sehr viel weniger“ oder „weniger" angegeben werden. Zudem wird auch die Zeitspanne, die die Teilnehmer:innen unter natürlichem Tageslicht verbringen, kürzer. Sie zeigt sich bei $44,2 \%$ der Teilnehmer:innen als reduziert. Hingegen verbringen die Kinder wie auch Jugendlichen wesentlich mehr Zeit mit Smartphone, Tablet oder PC (85,0\%). Unter diesen Umständen hat sich auch das Schlafverhalten seit Corona unter den Kindern und Jugendlichen stark verändert.

Mehr als jedes drittes Kind (37\%) gibt an, seit Corona eine schlechtere Schlafqualität aufzuweisen. 38,9\% der Kinder berichten sogar, nun Probleme mit dem Schlaf zu haben, was für dieses junge Alter (6-18 Lj.) äußerst unüblich ist. Von denjenigen, die über diese Schlafprobleme berichten, sind $42,5 \%$ von Einschlafschwierigkeiten und $20,3 \%$ von Durchschlafschwierigkeiten geplagt. Außerdem gehen 9 von 10 Kindern/Jugendlichen $(94,3 \%)$ nun während der Woche später zu Bett. Altersspezifisch zeigen sich die meisten 
Corona-bedingten Schlafprobleme bei den Jugendlichen (45,3\%) und eine Häufung von Albträumen bei den Jüngsten (16,3\%).

263

264

265

266

267

268

269

270

271

272

273

274

275

276

277

278

279

280

281

282

283

284

285

286

287

288

289

290

291

292

293

294

295

296

297

\section{Resümee}

Zusammenfassend zeichnet sich ein alarmierendes Bild der psychosozialen Gesundheit bei Kindern und Jugendlichen ab und verdeutlich den akuten Handlungsbedarf. Die besonders eindringlichen und noch nicht veröffentlichten (anonymen) Kommentare der Kinder und Jugendlichen der "Jetzt Sprichst Du“-Umfrage zeichnen hier ein besonders deutliches Bild (siehe ${ }^{3}$ ).

Als sinnbildliche Handlungsempfehlung wollen wir "Bewegung und Begegnung" ausgeben. Leider sind es ausgerechnet die Faktoren, die den Kindern und Jugendlichen gut durch die Pandemie helfen würden, die aufgrund der Corona- Maßnahmen unmöglich gemacht werden oder nur sehr bedingt möglich sind.

Aus unserer Sicht muss es daher erste Priorität sein, sich um eine rasche Normalisierung inklusive des Freizeit- und Sport-Angebots für Kinder und Jugendliche zu bemühen und im Sinne der Prävention schon heute tätig zu werden. Die Auswirkungen des oft zitierten „verlorenen Jahres“ für Kinder und Jugendliche sind sowohl aus psychologischer als auch psychotherapeutischer Sicht in ihrem Ausmaß heute nur schwer abschätzbar. Es ist aber wohl unbestritten, dass sie massiv sein werden und sich sowohl in psychosomatischen Erkrankungen [9] als auch in Verhaltensauffälligkeiten wie Schulverweigerung widerspiegeln werden. In diesem Sinne wäre es auch angezeigt, schon heute an den Ausbau von schulpsychologischen wie auch pädagogischen und psychotherapeutischen Angeboten für Kinder und Jugendliche zu denken.

Persönlich befürchten wir, dass im psychosozialen Sektor das Schlimmste noch bevorstehen könnte. Dann nämlich - wenn sich die wirtschaftlichen Folgen der Pandemie inklusive erhöhter Arbeitslosigkeit zeigen und somit auch der Stress in den Familien steigt ist auch mit vermehrter Gewalt in Familien [10] und in der Folge mit psychischen Reaktionen der Kinder und Jugendlichen zu rechnen [11].

Aus unserer Sicht ist daher klar: Wir müssen heute handeln, um nicht morgen vor den Scherben unserer Versäumnisse zu stehen und eine „verlorene Generation“ verantworten zu müssen.

Literatur

1. Fegert JM, Vitiello B, Plener PL, Clemens V (2020) Challenges and burden of the Coronavirus 2019 (COVID-19) pandemic for child and adolescent mental health: a narrative review to highlight clinical and research needs in the acute phase and the long return to normality. Child Adolesc Psychiatry Ment Health 14(20). https://doi.org/10.1186/s13034-020-00329-3

\footnotetext{
${ }^{3}$ Siehe http://bit.ly/JetztSprichstDu supplements
} 
2. Langmeyer A, Guglhör-Rudan A, Naab T, Urlen M, Winkelhofer U (2020) Kind sein in Zeiten von Corona. Ergebnisbericht zur Situation von Kindern während des Lockdowns im Frühjahr 2020. Deutsches Jugendinstitut. https://www.dji.de/fileadmin/user upload/bibs2020/Ergebnisbericht Kindsein Corona 2020.pdf

3. Ravens-Sieberer U, Kaman A, Otto C, Adedeji A, Devine J, Erhart M, Napp AK, Becker M, Blanck-Stellmacher U, Löffler C, Schlack R, Hurrelmann K (2020) Mental health and quality of life in children and adolescents during the COVID-19 pandemic - results of the COPSY study. Dtsch Arztebl Int 2020; 117: 828-9. https://doi.org/10.3238/arztebl.2020.0828

4. de Figueiredo CS, Sandre PC, Portugal LCL, Mázala-de-Oliveira T, da Silva Chagas L, Raony Í, Ferreira ES, Giestal-de-Araujo E, Dos Santos AA, Bomfim PO (2021) COVID-19 pandemic impact on children and adolescents' mental health: Biological, environmental, and social factors. Prog Neuropsychopharmacol Biol Psychiatry. 2021 Mar 2; 106: 110171. https://doi.org/10.1016/j.pnpbp.2020.110171

5. Andresen S, Lips A, Möller R, Rusack T, Schröer W, Thomas S, Wilmes J (2020) JuCo - Erfahrungen und Perspektiven von jungen Menschen während der CoronaMaßnahmen. Universitätsverlag Hildesheim. https://doi.org/10.18442/120

6. Bothe K, Schabus M, Eigl E-S, Hoedlmoser K (2021) How do Austrian children and adolescents sleep during the COVID-19 pandemic? Results from the "Jetzt sprichst Du!" survey. Manuscript in preparation.

7. Clift A K, Coupland C A C, Keogh R H, Diaz-Ordaz K, Williamson E, Harrison E M et al. (2020) Living risk prediction algorithm (QCOVID) for risk of hospital admission and mortality from coronavirus 19 in adults: national derivation and validation cohort study. BMJ 2020; 371. https://doi:10.1136/bmj.m3731

8. Berner R, Walger P, Simon A, Fischbach T, Dötsch J, Schneider, D, Huppertz H-I, Hübner J (2021) Stellungnahme der Deutschen Gesellschaft für Pädiatrische Infektiologie (DGPI) und der Deutschen Gesellschaft für Krankenhaushygiene (DGKH) zu Hospitalisierung und Sterblichkeit von COVID-19 bei Kindern in Deutschland. (Stand April 2021) Mortalität Kinder 21042021 korr (3).tiff (dgpi.de)

9. Pieh C, Plener PL, Probst T, Dale R, Humer E (2021) Mental health in adolescents during COVID-19-related social distancing and home-schooling. SSRN Electronic Journal. https://doi.org/10.2139/ssrn.3795639

10. Lee SJ, Ward KP, Lee JY, Rodriguez CM (2021) Parental social isolation and child maltreatment risk during the COVID-19 pandemic. $J$ Fam Viol. https://doi.org/10.1007/s10896-020-00244-3

11. Ravens-Sieberer U, Kaman A, Erhart M, Devine J, Schlack R, Otto C (2021) Impact of the COVID-19 pandemic on quality of life and mental health in children and adolescents in Germany. Eur Child \& Adolesc Psychiatry. https://doi.org/10.1007/s00787-021-01726-5 